\title{
COOPERACIÓN Y NO-COOPERACIÓN ESTRATÉGICA: EFECTOS SOBRE LA PRODUCTIVIDAD Y LA COMPETITIVIDAD
}

Rafael E. Capellán, M.A.*

\section{RESUMEN}

La cooperación estratégica entre los actores de la cadena productiva se traduce en incrementos significativos en los niveles de ingresos y productividad de los mismos, así como del conglomerado en su totalidad. Una adecuada planificación estratégica que involucre los distintos componentes de la cadena de valor genera un mayor nivel de calidad del producto ofrecido y por ende contribuye a fortalecer la competitividad del conjunto productivo o cluster.

Si comparamos escenarios estratégicos-cooperativos con escenarios estratégicos no-cooperativos, tenemos que los indicadores de desempeño de los primeros exceden de manera sustancial los del segundo tipo. Es el objetivo del presente artículo determinar los beneficios reales de la cooperación estratégica, tanto inter como intra-actores, en contraposición al comportamiento natural de rivalidad y competencia que genera el posicionamiento de mercado. Mediante la utilización de las principales y más recientes teorías y modelos sobre el tema, demostraremos cómo, una vez creada la conciencia de los beneficios inherentes a la cooperación, las tradicionales prácticas individualistas de producción y competencia pura tienden a perder validez.

* El autor es Economista con una maestría en Estudios de Desarrollo. Actualmente se desempeña como Coordinador de Proyectos del Consejo Nacional de Competitividad CNC. 
Cooperación estratégica, rivalidades, competencia, productividad, competitividad, crecimiento económico.

\section{Base Teórica del Concepto de Cooperación}

$\mathrm{Al}$ hablar de cooperación resulta importante hacer referencia a los cimientos teóricos que sustentan el término y que por tanto ofrecen una explicación racional al alcance del mismo en la práctica. Así tenemos que la cooperación, entendida como la sinergia entre dos o más actores, tiene como meta principal el obtener la mayor cantidad de beneficios para el grupo a partir de una situación o escenario dado, en nuestro caso específico, a partir del mercado.

Iniciaremos nuestro análisis utilizando la terminología del matemático húngaro John von Neumann (1903-1957): "juegos de suma cero" (zero sum games) y "juegos de no-suma cero" (non-zero-sum games). Los primeros se refieren a juegos de dos actores en los cuales el monto de recursos obtenibles es fijo y por tanto la ganancia de una de las partes representa necesariamente una pérdida del otro lado, en otras palabras, estamos hablando de competencia pura. Asumiendo las ganancias como positivas y las pérdidas como negativas, la suma de ambas variables arrojará siempre el mismo resultado: cero (Holt, 2004).

$$
\begin{aligned}
& \underset{\mathrm{G}=0}{\mathrm{q}}(\mathrm{G} a+\mathrm{Gb})=\sum_{\mathrm{P}=0}^{\mathrm{q}}(-\mathrm{Pb}-\mathrm{Pa}) ; \underset{\mathrm{G}=0}{\mathrm{q}}(\mathrm{Ga}+\mathrm{Gb})+\sum_{\mathrm{P}=0}^{\mathrm{q}}(-\mathrm{Pb}-\mathrm{Pa})=0 \\
& \text { Ga }: \text { Ganancias actor }(a) \\
& \text { Gb }: \text { Ganancias actor }(b) \\
& P a: \text { Pérdidas actor }(a) \\
& P b: \text { Pérdidas actor }(b)
\end{aligned}
$$


Por otro lado, los juegos de no-suma cero se relacionan directamente con el citado concepto de cooperación, ya que la cantidad de bienes o recursos obtenibles por las partes es variable y se pueden generar acuerdos formales entre los actores con el propósito de obtener mayores beneficios para el conjunto.

A su vez el premio Nóbel de Economía de 1994, el norteamericano John Nash, elaboró su trascendental equilibrio ampliando la noción y el alcance de los juegos de no-suma cero de Neumann, mediante la introducción de un escenario de dos o más actores en el cual no existe cooperación estratégica y donde las estrategias individuales están influenciadas por las expectativas de las estrategias del resto del grupo. En este caso el equilibrio se alcanza cuando cada actor aplica aquella estrategia que le proporciona el mayor beneficio posible tomando en cuenta las estrategias a aplicar por los demás actores, todo esto bajo condiciones de competencia y rivalidad. Este enfoque aunque ha revolucionado la teoría económica hasta nuestros días, igual presenta algunas limitantes relacionadas con temas de comportamiento irracional, comunicación, información incompleta, justicia social, ética, etc. (Ver Myerson, 1999). Es aquí donde entra nuevamente el tema de la cooperación real entre actores económicos.

En el contexto del equilibrio de Nash la noción de nocooperación se sustenta en una especie de "acuerdo forzado" donde cada actor no necesita de un agente externo que legitimice la aplicación del mismo, sino que la expectativa de beneficios "optimizados" por parte de los involucrados es suficiente para hacer tácito el "convenio" vía la implementación de las estrategias individuales (Holt, 2004). Partiendo de esta interpretación de roles, la cooperación implica necesariamente la existencia de un agente o instancia que se 
encargue de legitimizar ${ }^{2}$ los acuerdos y estrategias asumidas por las partes. De hecho esta es la forma en que se lleva a cabo en la práctica.

Cimentándonos en las citadas teorías precursoras de von Neumann y Nash podemos afirmar que la cooperación implica el uso de estrategias comunes y formales encaminadas hacia un mismo fin: obtener mayores beneficios para el grupo en su conjunto; dejando por tanto de lado los modelos tradicionales de competencia individualista entre actores similares de una misma cadena. En este último caso el beneficio de cada actor implica una pérdida por parte de sus competidores. La nueva tendencia a analizar consiste en el abandono de la competencia a lo interno de la cadena o grupo productivo y su transferencia al ámbito sectorial, regional o internacional, mientras se generan incrementos notables en los niveles de productividad y competitividad de los grupos. Posteriormente haremos referencia a otras teorías y modelos más contemporáneos sobre el tema de la cooperación entre agentes, específicamente al modelo de clusters.

A lo largo de las líneas subsiguientes pretendemos demostrar cómo los beneficios obtenidos por un conjunto de actores bajo condiciones de cooperación estratégica son mayores que los resultados individuales obtenidos bajo condiciones de no-cooperación, incluso en caso de equilibrio. Este hecho ha sido magistralmente ilustrado a partir del análisis de los matemáticos Melvin Dresher y Merrill Flood, quienes demostraron a partir del famoso "Dilema del Prisionero" cómo los actores obtienen mayores beneficios cuando cooperan

2 Esta legitimación no necesariamente implica el uso de las estructuras jurídicas formales, sino más bien constituye un acuerdo de entendimiento asumido y registrado por las partes, bajo la coordinación de una unidad neutra (asociación, cámara, federación, ONG, etc.) 
con relación a la escogencia de una posición de equilibrio nocooperadora (Holt, 2004).

\section{Competitividad y Productividad: Motores del Crecimiento Económico}

En los últimos años el tema de la Competitividad ha ocupado una posición de primer orden en la agenda internacional para el crecimiento económico y la lucha contra la pobreza. Importantes escuelas internacionales de negocios y desarrollo tales como Harvard han destinado cuantiosos recursos a la investigación en el área. Y es que la competitividad entendida como la capacidad de un país, empresa o grupo de empresas de posicionarse exitosamente en el entorno de los negocios globales, juega un papel protagónico en los actuales tiempos de integración económica y apertura comercial.

A su vez el tema de la competitividad incluye como parte integral del mismo el concepto de "Cluster", o conglomerado productivo por su traducción más fiel al español, tan en boga dentro del discurso de la planificación estratégica y la gerencia contemporánea. Según el padre de la competitividad moderna, el profesor Michael Porter, el cluster no es más que una concentración geográfica de compañías, suplidores especializados, proveedores de servicios, industrias relacionadas e instituciones asociadas, todas interconectadas en un área productiva en particular y que compiten pero que al igual cooperan entre si. Es precisamente a partir de este modelo productivo como las economías más avanzadas han logrado dar respuesta eficiente a los retos presentados por un entorno de negocios cada vez más complejo y dinámico, definido por la transferencia de información y la competencia basada en resultados y calidad (Porter, 1998). 
Tomando en cuenta el carácter eminentemente dinámico de los mercados internacionales, el cluster confiere una ventaja crucial a los productores, ya que les permite "reinventarse" a sí mismos, adaptándose a las variaciones en los gustos y preferencias de los consumidores vía la adecuación tecnológica y la diversificación productiva (Duch, 2002). La herramienta de la asociatividad empresarial como elemento homólogo al concepto de cooperación, tiene una influencia directa en el crecimiento económico y en la generación de riqueza de los países y de las firmas, sin embargo es necesario introducir una variable imprescindible dentro del análisis de la competitividad empresarial, la productividad.

La realidad es que competitividad y productividad son conceptos análogos y complementarios a la vez: el uno implica al otro. Los países y las firmas más competitivas del mundo son igualmente las de mayor productividad en sus respectivas áreas. El World Economic Forum establece en su Informe Global de Competitividad dos índices principales por país, estos son: el índice de Competitividad del Crecimiento y el índice de Competitividad Microeconómica. El primero comprende los resultados macro del crecimiento de las economías, tomando en cuenta el desempeño económico del país, su innovación tecnológica, la eficiencia de sus instituciones públicas y las variaciones de sus indicadores macroeconómicos en general. Por su parte el segundo índice se centra en los indicadores a nivel de las empresas tales como: la gestión de operaciones, el manejo estratégico, la calidad del clima de negocios e inversiones y el producto interno bruto per cápita. No resulta entonces casual que las economías de mayor producción e ingreso por número de habitantes sean las más competitivas a nivel mundial. Así tenemos que naciones como los EEUU, Finlandia, Reino Unido, Alemania y Suiza ocupan los primeros lugares en el ranking, denotando la inexorable relación entre competitividad, crecimiento económico y 
productividad. Implícitamente el fenómeno que nos compete sobre la cooperación entre agentes, queda reflejado en el índice de competitividad microeconómica. (Ver World Economic Forum, 2004).

Según el citado informe, los fundamentos microeconómicos de la productividad son el nivel de tecnicidad y refinamiento con que compiten las compañías domésticas y extranjeras que operan en el país, así como la calidad del clima de micro-negocios en el cual se desenvuelven. La productividad guarda una estrecha relación con la capacitación del recurso humano, la disponibilidad de información, la eficiencia de los procesos gubernamentales, la calidad de la infraestructura, entre otros factores. Un indicador bastante simple y fiable del grado de productividad de una economía lo es su PIB per cápita o su PIB sobre la población económicamente activa (PIB/PEA). Luego a nivel micro (por unidad empresarial o por cluster) se pueden utilizar los ingresos totales de la unidad o grupo, divididos por su capital de trabajo $(\mathrm{I} / \mathrm{K})$ y por su nivel de empleo productivo (I/L). Para el caso del presente análisis, y debido a limitantes relativas al acceso de información particular de firmas o grupos productivos, optaremos por investigar los cambios en términos de rendimiento económico de aquellos sectores que han demostrado tener un mayor nivel de asociatividad y/o cooperación entre sus agentes, y aquellos que han optado por esquemas operativos más individualistas a nivel de sus actores.

Para el caso específico dominicano es importante hacer notar que el modelo de clusters, el cual fomenta la asociatividad y la cooperación entre actores, está siendo implementado por un creciente número de firmas y de productores particulares, arrojando hasta el momento resultados realmente asombrosos. Tal es el caso de la industria turística y algunos segmentos de la agroindustria no-tradicional (productos étnicos). En términos generales podemos decir que República Dominicana en su condición de país de mediano ingreso presenta índices globa- 
les de competitividad bastante halagüeños con relación a otros países de América Latina tales como: Argentina, Uruguay, Perú, Venezuela, El Salvador y Guatemala, los cuales presentan mayores o similares niveles de desarrollo y capacidad productiva. De hecho RD ocupaba hacia el año 2002 la posición número 38 dentro del ranking de crecimiento económico de 128 países a nivel mundial (Vial, 2002).

\section{Cooperación Estratégica: Clave del Éxito}

Con el objetivo de ilustrar los efectos positivos de la cooperación estratégica sobre la competitividad y el crecimiento económico, nos disponemos a realizar un análisis comparativo bastante puntual sobre las variaciones del producto interno bruto y de las productividades de los sectores turismo y agroindustria, en contraposición a sectores productivos con rasgos incipientes de cooperación en sus respectivas cadenas de valor, pero que igual operan bajo condiciones propias de la competencia pura (muchos competidores intentando obtener la mayor tajada posible del mercado), entre estos: servicios financieros, comunicaciones, construcción y comercio. Igualmente hemos incluido en el análisis otros sectores que operan bajo condiciones cercanas al oligopolio tales como: minería, producción azucarera y electricidad y agua, los cuales experimentan un desincentivo a la cooperación inter-agente debido a los ingresos más o menos constantes y a un mercado semi-cautivo. Así podremos ver cómo las tasas de crecimiento del PIB y de la razón PIB/PEA sectorial para el período 1996-2003, varían en función del nivel de cooperación estratégica existente, pudiendo igual verse afectadas por factores exógenos tales como la inversión.

Es importante señalar que la escogencia de los sectores: Turismo y agroindustria como aquellos de mayor grado de cooperación y asociatividad entre sus actores, se debe a la exis- 
tencia de ejemplos tangibles de conglomerados productivos en dichas ramas de producción, y entre los cuales podemos citar: Los clusters turísticos de Romana-Bayahibe y Puerto Plata, el cluster eco turístico y el hortofrutícola de La Vega, el cluster de mango de Moca y el cluster de café a nivel nacional, entre los principales. Estos grupos han logrado incrementar sustancialmente sus ingresos por concepto de ventas y de exportaciones, situándose muy por encima de otros actores y productores similares en sus respectivos sectores, pero que no han optado por el sugerido modelo de asociatividad y cooperación.

Así pues, hemos clasificado los sectores de acuerdo a su nivel de asociatividad empresarial, medida a través de la existencia de clusters o grupos estratégicos, así como de agendas comunes entre los actores.

\section{Cuadro 1}

Producto Interno Bruto para los Sectores Seleccionados Período 1996-2003

\begin{tabular}{|c|c|c|c|}
\hline \multirow{2}{*}{ SECTORES } & \multicolumn{2}{|c|}{ PIB } & \multirow{2}{*}{$\begin{array}{l}\text { T.C } \\
(\%)\end{array}$} \\
\hline & 1996 & 2003 & \\
\hline \multicolumn{4}{|c|}{ Sectores que presentan indicios de cooperación estratégica } \\
\hline Agricultura & 313.9 & 360.9 & 15.0 \\
\hline Hoteles, Bares y Restaurantes & 292.6 & 574.2 & 96.2 \\
\hline \multicolumn{4}{|c|}{$\begin{array}{l}\text { Sectores de alto crecimiento con indicios incipientes } \\
\text { de cooperación estratégica }\end{array}$} \\
\hline Construcción & 501.6 & 828.4 & 65.2 \\
\hline Comercio & 603.9 & 813.3 & 34.7 \\
\hline Comunicaciones & 185.7 & 695.3 & 274.4 \\
\hline Electricidad y Agua & 96.3 & 163.0 & 69.3 \\
\hline \multicolumn{4}{|c|}{ Sectores que no presentan indicios de cooperación estratégica } \\
\hline Minería & 128.6 & 111.0 & -13.7 \\
\hline Azúcar & 47.5 & 40.6 & -14.5 \\
\hline Finanzas & 228.8 & 265.9 & 16.2 \\
\hline
\end{tabular}

Fuente: Banco Central de la República Dominicana

Nota: El PIB está expresado en millones de RD\$ a precios de 1970 
A partir del cuadro 1 podemos constatar que los sectores que han manifestado la existencia de eficientes redes de cooperación y altos niveles de asociatividad entre los actores de sus respectivas cadenas productivas (aún de manera incipiente), han logrado tasas de crecimiento superiores a los sectores que no presentan en absoluto indicios de cooperación estratégica, con la excepción del sector finanzas. Para este último caso es importante hacer notar que a pesar del crecimiento en términos absolutos del PIB del sector finanzas, su productividad decrece notablemente para el mismo período como podremos ver en el cuadro 2. En cuanto al caso de agricultura, su tasa de crecimiento no ha sido mayor debido a la imposibilidad de aislar el PIB para aquellos grupos de productores no-tradicionales, quienes son los que efectivamente han logrado un éxito impresionante en términos de sus ventas y exportaciones debido al cambio de mentalidad de sus miembros y a la adopción del modelo productivo de cluster (e.g. Cluster Hortofrutícola de La Vega).

\section{Cuadro 2}

Productividad por Sector Seleccionado (Producto Interno Bruto entre Población Económicamente Activa PIB/PEA )

Período 1996-2003

\begin{tabular}{|l|l|l|l|}
\hline \multirow{2}{*}{ SECTORES } & \multicolumn{2}{|c|}{ PIB/PEA } & \multirow{2}{*}{$\begin{array}{c}\text { T.C. } \\
(\%)\end{array}$} \\
\cline { 2 - 3 } & \multicolumn{1}{|c|}{1996} & 2003 & \multicolumn{1}{|c|}{$(\%)$} \\
\hline \multicolumn{3}{|c|}{ Sectores que presentan indicios de cooperación estratégica } \\
\hline Agricultura & 0.06 & 0.082 & 37.0 \\
\hline Hoteles, Bares y Restaurantes & 0.209 & 0.279 & 33.5 \\
\hline \multicolumn{3}{|c|}{ Sectores de alto crecimiento con indicios incipientes } \\
de cooperación estratégica \\
\hline Construcción & 0.263 & 0.334 & 27.0 \\
\hline Comercio & 0.112 & 0.113 & 1.1 \\
\hline Comunicaciones & 0.29 & 0.442 & 52.6 \\
\hline
\end{tabular}




\begin{tabular}{|l|l|l|l|}
\hline Electricidad y Agua & 0.63 & 0.611 & -3.0 \\
\hline \multicolumn{2}{|c|}{ Sectores que no presentan indicios de cooperación estratégica } \\
\hline Minería & 1.35 & 1.453 & 7.7 \\
\hline Azúcar & N/D & N/D & N/D \\
\hline Finanzas & 0.55 & 0.364 & -34.4 \\
\hline
\end{tabular}

Fuente: Banco Central de la República Dominicana

Nota: Los índices de productividad están expresados en millones de RD\$ a precios de 1970 sobre la PEA sectorial $* 100$

Cuando contrastamos los resultados del cuadro 1 con los del cuadro 2 observamos que la tendencia se mantiene, esto es, la productividad de los sectores que operan bajo esquemas de cooperación estratégica crece en mayor proporción que la productividad de los sectores que no lo hacen. Con respecto a los cuatro sectores que presentan evidencia limitada de esquemas de cooperación estratégica (construcción, comercio, comunicaciones y electricidad y agua) podemos decir que los mismos, a pesar de haber logrado tasas muy favorables de crecimiento en sus PIB, básicamente debido a factores exógenos tales como: incrementos en los niveles de inversión local y extranjera, así como al aumento significativo de la demanda local para estos renglones, sus productividades individuales crecen en mucho menor proporción, e incluso llegan a decrecer para el caso de electricidad y agua (adicionando la naturaleza oligopólica de este sector). Sólo el sector de las comunicaciones presenta un crecimiento en su productividad mayor al experimentado por los sectores de turismo y agricultura.

Igualmente el buen desempeño de estos cuatro sectores puede estar relacionado con la implementación de planes estratégicos a lo interno de sus unidades productivas (juegos de no-cooperación estratégica) específicamente para el caso de las comunicaciones. No obstante, la sostenibilidad del creci- 
miento en el tiempo y el efecto multiplicador del mismo en las comunidades y agentes regionales relacionados a la cadena, sólo se logrará mediante la cooperación estratégica inter-agente y no exclusivamente vía la planificación individual (Lotero, 1995). Es por esto por lo que observamos menores niveles de productividad para las referidas actividades con relación a turismo y agroindustria, aunque de cualquier manera no descartamos un desarrollo incipiente (y en expansión) para los esquemas de cooperación de estos sectores, ya que los mismos cuentan con excelentes canales de comercialización y mantienen un estrecho vínculo con sus suplidores y con las instituciones de apoyo en sus respectivas áreas.

El éxito de la cooperación estratégica reside pues en el cambio del paradigma productivo tradicional basado en la competencia pura individualista, hacia un modelo operativo donde se busca la colaboración y el compromiso legítimo de los distintos actores de la cadena de valor con el fin de optimizar procesos, reducir costos, incrementar la calidad del producto y obtener mayores beneficios para todos (ver Okko, 2003). Este cambio, sin embargo, no es una tarea fácil. Aún existe una gran resistencia de parte de los círculos productivos en asumir un esquema basado en el flujo libre de información entre actores, "cost-sharing" en la implementación de estrategias y proyectos conjuntos, así como una distribución consensuada de los beneficios e ingresos proyectados. Una solución viable a esta problemática sería el modelo estratégico de "subir las apuestas", o "raising-the-stakes" (RTS) estudiado por los profesores ingleses Roberts y Renwick; dicho modelo plantea que los actores de la cadena realicen inversiones mínimas al principio de la relación, y luego, dependiendo de la colaboración de los demás miembros, ir incrementando los montos y reduciendo los riesgos en forma gradual, mientras se fortalece y desarrolla la dinámica de reciprocidad de los involucrados (Roberts, 2003). 
De todos modos, e independientemente de estos obstáculos ideológicos remanentes, cada vez más se crea la conciencia de una interdependencia ineludible entre las firmas modernas, las instituciones públicas, y los actores complementarios de la cadena, tanto a nivel sectorial como geográfico (incluyendo los planos regional e internacional). La conquista de la competitividad requiere pues de una real transformación de conocimientos cimentada en un adecuado y necesario cambio técnico-institucional. Todo esto implica a su vez un profundo cambio de mentalidad por parte de los actores, el cual puede ser catalizado vía el desarrollo de nuevos procesos de aprendizaje y de creación de competencias técnicas (Ver Lotero, 1995 y Yoguel, 2000).

Una vez iniciado el referido cambio se espera un efecto directo sobre los niveles de calidad en los procesos y productos generados por los grupos y las firmas. La calidad constituye un elemento central en el camino hacia la competitividad, sobre todo considerando que en el actual modelo económico internacional la relación precio/valor es un criterio fundamental en las decisiones de compra. Los procesos de normalización, metrología, certificación y acreditación resultan vitales para la gestión de la calidad. Así tenemos importantes sistemas internaciones de acreditación de normas, tales como el ISO, los cuales constituyen requisitos indispensables en el marco de los crecientes acuerdos de comercio tanto regionales como bilaterales. Esto a su vez implica un esfuerzo conjunto sector público y sector privado en aras de materializar las inversiones necesarias en materia de laboratorios y organismos especializados sobre temas de metrología y acreditación. Todo este proceso se viabiliza notablemente a partir de la cooperación entre agentes y el citado modelo de asociatividad. 


\section{Coo-petencia: Su Rol en el Desarrollo Productivo}

Una vez abordados los conceptos de competencia pura, cooperación estratégica y competitividad desde la perspectiva del modelo de clusters, damos paso a un nuevo concepto derivado a partir de todos los anteriores, este es el concepto de la "Coo-petencia". El mismo surge de la fusión etimológica: cooperación + competencia, y no es más que una innovadora estrategia de negocios que aglutina los beneficios de ambas nociones integrantes mientras prioriza el desarrollo de la industria, sector, o grupo productivo sobre el beneficio particular de la firma.

Dicha metodología implica un buen nivel de fluidez operativa a lo interno de las redes de valores donde convergen: firmas, clientes, proveedores y competidores, así como la interacción de los mismos para el diseño de planes estratégicos encaminados a incrementar los beneficios totales del grupo. Sin embargo, la red o conjunto productivo no se limita a estos cuatro actores principales. A partir del análisis de Nalebuff surge un interesante elemento que viene a enriquecer aún más el modelo de conjunto productivo, este es: el agente "complementador"3; o sea aquella firma que suministra productos y servicios complementarios a la oferta principal del resto de la industria o grupo ${ }^{4}$ (Nalebuff, 1996).

Si además del complementador adicionamos aquellas instituciones y elementos de soporte productivo, tales como: institutos de formación técnico-profesional, entidades financieras

3 Este elemento ha sido incluido por Porter bajo la categoría de empresas de apoyo (ver Porter, 1998).

4 Un buen ejemplo sería el vendedor de artesanías que opera en el marco de la industria hotelera y quien no califica como competidor ni como proveedor de la misma, esto dado que su actividad más bien complementa el producto central (digase: hospedaje, alimentación, diversión, etc.) 
especializadas, proveedores de servicios públicos (autoridades locales y ayuntamientos), obras de infraestructura física, etc. tenemos entonces que la coo-petencia implica mucho más que la interrelación: firmas-clientes-proveedores. Estamos hablando de un sistema bastante articulado que amerita de una comunicación fluida y una agenda común de acción. Sólo mediante la eficiente integración y colaboración de todos los actores de la cadena podremos alcanzar los resultados esperados.

Dada la complejidad de las redes productivas y la vital interacción entre sus distintos componentes, la confianza, la creación de competencias, y el trabajo en equipo, resultan elementos imprescindibles para la puesta en marcha y el éxito del modelo. Igualmente no se pueden dejar de lado los aspectos relativos a la responsabilidad social y la ética empresarial, ya que los mismos tienen una incidencia directa sobre el entorno en el cual operan las unidades. Como pudimos ver a partir de las mediciones de los índices globales de competitividad del World Economic Forum, el ambiente de micro-negocios constituye un elemento crucial de cara a la productividad y el desempeño de los actores.

Los preceptos de interacción anteriormente tratados a lo interno de los grupos y entre sus enclaves integrales, también deben ser aplicados tanto a la estructura individual de cada firma; esto desde el punto de vista de la confianza y el compromiso de sus empleados; como también hacia lo externo en la sociedad, sobre todo en lo relativo a la ética y la confianza pública generada por la unidad productiva. En este sentido existe una estrecha correlación entre la prosperidad del grupo, la prosperidad de la unidad de producción con sus respectivos integrantes y por ende la prosperidad de la sociedad civil en su conjunto; de ahí la importancia de la confianza y la coo-petencia en el desarrollo de las redes productivas (Ver Osuna, 2002). 
Luego tenemos los elementos de trabajo en equipo y creación de competencias como otros dos enclaves fundamentales dentro del proceso de optimización de eficiencia y productividad compartida. Por su parte Franco y Velásquez presentan un esquema de beneficios mutuos sustentado en la responsabilidad, las habilidades y el compromiso del equipo humano, e igual plantean las variables de seguridad, (en términos de protección de amenazas comunes), y la autoestima, (en términos de reconocimiento de la contribución individual), como elementos cruciales para la consecución de un desempeño mejorado por parte de las redes productivas (Franco, 2000). Una vez más la lógica operativa ofrecida por el modelo justifica el abandono de las prácticas tradicionales de competencia pura, de cara a un sistema mucho más cohesionado pero a la vez sensible y receptivo a los cambios en la dinámica de los negocios.

Finalmente, entendemos que la conquista de la competitividad amerita un proceso de transformación, más que nada ideológico, y que sin la misma la sostenibilidad del crecimiento de las distintas economías del mundo puede verse seriamente afectada, sobre todo en los actuales tiempos de internacionalización comercial. Hemos visto como la cooperación estratégica guarda una estrecha relación con los niveles de ingreso y productividad de los grupos y firmas, por cuanto la adopción de modelos de asociatividad productiva resulta altamente atractiva para los fines del desarrollo internacional y la reducción en los niveles de pobreza. Por su parte la confianza de los agentes económicos y la creación de nuevas capacidades técnicas acorde a los cambios en las demandas del mercado, juegan un papel de primer orden en el discurso de la competitividad y el desarrollo productivo. Si deseamos alcanzar un adecuado nivel de bienestar socio-económico para nuestros países, debemos iniciar cuanto antes los cambios estructurales necesarios para dinamizar nuestros aparatos productivos y propiciar su exitosa participación en la danza económica mundial. 


\section{Bibliografía}

Banco Central de la República Dominicana (2004) "Mercado de Trabajo e Informes de la Economía Dominicana 1996 y 2003". <http:/www.bancentral.gov.do/infeco.html>

Duch, Emiliano (2002) "La Otra Cara de la Competitividad", Revista INCAE 11(3):56-57.

Franco, Carlos Alberto y Francisco Velásquez V. (2000) “ ¿Cómo mejorar la eficiencia operativa utilizando el trabajo en equipo?", Estudios Gerenciales (76):27-35, jul.-sep., 2000.

Holt, Charles A. and Alvin E. Roth (2004) "The Nash Equilibrium: A Perspective". <http://faculty-gsb.stanford.edu/wilson/E608_2004/ pdf\%20files/Basic\%20Game\%20Theory/Holt.Roth-Perspective. pdf>

Lotero C., Jorge ( 1995) "Industria, Estrategias y Competitividad Regional en el Occidente Colombiano“", Lecturas de Economía (42):71-105, ene.-jun., 1995

Myerson, Robert B. (1999) "Nash Equilibrium and the History of Economic Theory". Journal of Economic Literature 36: 1067-1082.

Nalebuff. Barry J. y Adam M. Brandenburger (1996) Coo-petencia. Bogotá: Norma.

Okko, Paavo (2003) "Competition and Cooperation", presentation at COGECA Conference, Turku School of Economics and Business Administration. Turku, Finland, November $13^{\text {th }}$.

Osuna F., Carlos y Santiago Macías H. (2002) "La Confianza como base de la competitividad de las empresas", El mercado de Valores 62 (4) 31-6, abr., 2002

Porter, Michael E. (1998) On Competition. Harvard Business Review Book Series. Boston: Harvard Business School Press.

Roberts, Gilbert and James S. Renwick (2003) "The Development of Cooperative Relationships: An Experiment", Proceedings of the Royal Society London B (2003) 270, 2279-2283. <http://www. staff.ncl.ac.uk/gilbert.roberts/Roberts\&Renwick2003.pdf>

Vial, Joaquín (2002) “Cuán Competitiva es la República Dominicana?". Documentos de Trabajo. Center for International Develo- 
pment at Harvard University y Fundación Global Democracia y Desarrollo, Cambridge, USA, Diciembre 2002.

World Economic Forum (2004) "Global Competitiveness Reports 2002-2003 / 2003-2004". <http://www.weforum.org/site/homepublic.nsf/Content/Global+Competitiveness+Programme\%5CGloba 1+Competitiveness+Report>

Yoguel, Gabriel (2000) "Creación de Competencias en Ambientes Locales y Redes Productivas", Revista de la CEPAL (71):105-119, ago., 2000. 